\title{
SPH simulation of Periodic Wave Breaking in the Surf Zone - A Detailed Fluid Dynamic Validation -
}

\author{
Authors \\ Riccardo Angelini Rota Roselli ${ }^{1,3}$, Giuliano Vernengo ${ }^{2}$, Stefano Brizzolara ${ }^{3}$ and Roberto Guercio ${ }^{1}$ \\ ${ }^{1}$ Civil and Environmental Engineering Department, DICEA, La Sapienza University, Via Eudossiana 18, 00184, Rome, Italy \\ ${ }^{2}$ Naval Architecture Department, DITEN, University of Genova, Via Montallegro 1, 16145, Genoa, Italy \\ ${ }^{3}$ Aerospace and Ocean Engineering Department, Virginia Tech, 460 Old Turner Street, Blacksburg, 24061, VA, USA \\ email: riccardo.angelinirota@uniroma1.it; giuliano.vernengo@unige.it; stebriz@vt.edu, roberto.guercio@uniroma1.it
}

\begin{abstract}
The estimation of wave breaking and run-up on sloped beaches is a relevant issue in different coastal engineering applications. The present study stresses on the capabilities of a Smoothed Particle Hydrodynamics (SPH) solver, with optimal numerical and physical parameters, to accurately simulate the complex flow field in the surf zone and run-up region. Numerical results are compared with high quality experimental measurements of the local flow field in terms of instantaneous and phase averaged values. The selected test case regards the propagation and breaking of regular non-linear waves on a smooth impermeable plane slope. The comparison is based on a complete set of 128 consecutive non-linear regular waves. The level of accuracy of the numerical results and the ability of the model to reproduce the periodic flow in the surf-zone is provided. Current limitations and uncertainty sources are identified and discussed to guide future developments.
\end{abstract}

Keywords: Surf-Zone wave transformation, Waves Breaking, Waves Shoaling, Run-Up, Smoothed Particle Hydrodynamics (SPH)

\section{Introduction}

Achieving a reliable prediction of the wave transformations in the surf-zone is extremely relevant in many coastal engineering applications. In fact, the design of wave-breakers, sea walls and other structures might change based on the occurrence of these wave modification processes. They also affect coastal risk-maps since e.g. flooding phenomena on some areas might be strongly connected to wave run-up on beaches. Despite several theoretical and approximated models have been developed in the past years such hydrodynamics phenomena are characterized by strong non-linear modification of the free surface that increase the complexity of the solutions that cannot be easily described by simple formulations. Keeping this in mind the only viable alternative to expensive model tests is the numerical modeling of the problem by ad-hoc developed and calibrated Computational Fluid Dynamics (CFD) models. The ability of predicting the types and position of breaking and the consequent energy losses of flow field is important to outline coastal risk-maps or to predict sediment transport and manage erosion in sandy shorelines. Many studies have greatly improved the knowledge of the hydrodynamics in the surf zone (see for instance Granthem (1953), Saville Jr (1955), Saville (1956, 1958); Savage (1959)) and several empirical formulations and correlation curves have been developed based on the results of laboratory experiments. Thompson (1989) and Kobayashi et al. (1987) introduced approximate theoretical models for the prediction of the characteristics of regular waves breaking over impermeable slopes. In the past two decades a variety of theoretical and numerical methods have been developed to simulate run up of periodic and, more frequently, solitary waves on sloped beaches. However, the accuracy in simulating complex wave phenomena such as breaking and propagation on sloped bottom is still a matter of investigation (Martinez-Ferrer et al., 2018). A main issues is to achieve reliable repeatability of the periodic breaking phenomenon, both in the numerical and physical domain. This is related to the intrinsic unstable nature of the wave breaking phenomenon, which ends up being 
very sensitive to stochastic disturbances, and to the difficulty of finding high quality detailed flow field measurements for periodic wave breaking. Approximate indications based on statistical interpretation of available empirical and numerical results are available to coastal engineers from various sources: design nomograms for wave breaking and run-up were provided since the earliest version of the Shore Protection Manual Army Corps of Engineers (1984) and in the Manual for Wave Runup Analysis (FEMA, 1981), derived from the early studies of Dean (1974), Weishar and Byrne (1978) and Stoa (1978). Some further improvements concerning the reanalysis of non-linear regular waves run-up experiments (e.g. Kobayashi (1999), Hughes (2004), and Hsu et al. (2012)) have been included in other design manuals and in several other publications but the high uncertainty affecting these approximate methods limit their applicability to preliminary design only. In this context, the need of high fidelity modeling tools for the simulation of shallow water waves transformation close to shore, is evident. The increase of computational efficiency (see e.g. Valdez-Balderas et al. (2013)) combined to the development and the continuous improvement of different CFD solvers for coastal waves modeling (e.g. Zijlema et al. (2011), Gomez-Gesteira et al. (2012), Higuera et al. (2013a,b), Bayon et al. (2016) and De Leffe et al. (2010)) offer new opportunities in this respect. Hydrodynamics of the surf zone requires the solution of a relatively broad range of characteristic scales: from the wave length to the turbulent vortexes induced by breaking. A Boussinesq-type approach has been used e.g. by Madsen et al. (2006) and by Bingham et al. (2009) while Ducrozet et al. (2012) used a High Order Spectral (HOS) method to develop a numerical wave tank. By means of both latter approaches the breaking of the wave is not directly simulated but it is modeled by using empirical formulations describing the wave height decreasing due to breaking. Current capabilities of fully non-linear Boussinesq models for shoaling processes using explicit or hybrid models is well reviewed in (Kirby, 2016). The empiricism required to introduce the effects of wave breaking in the Boussinesq reduced order model is the weakest aspect of these methods and introduces a certain degree of uncertainty. Navier-Stokes equation solvers (Higuera et al., 2013b) can be used to reproduce shoaling processes within the engineering accuracy. Large Eddy Simulation (LES) has been applied by to the same set of experimental data used in the present study by Lubin et al. (2011) and by Lubin and Glockner (2015) to analyze the air entrapment on plunging breakers in three dimensions.

This paper deals with the accuracy and consistency of
Smoothed Particle Hydrodynamics (SPH) solvers for long time simulations of periodic shallow water waves breaking over an impermeable slope. Long time repeatability is of the essence here. SPH flow solvers has been used for different studies ranging from wave propagation (De Chowdhury and Sannasiraj, 2013) to other non-linear free surface flows such as wave overtopping (Gomez-Gesteira et al., 2005) or interactions with coastal structures (De Chowdhury et al. (2017) and Didier et al. (2014)). This work contributes to the validation of very non- linear flow models, dealing with periodic wave breaking, fragmentation and sprays, The model is built upon the general purpose SPH solver implemented in the open-source code DualSPHysics (Gomez-Gesteira et al., 2012), which provides GP- GPU acceleration, hence allowing for highly resolved flow simulations (Vacondio et al., 2014). This SPH solver has been derived from the original solver SPHysics (Dalrymple and Knio, 2001), already used and validated for the prediction of solitary waves breaking of (Rogers and Dalrymple, 2005, 2008). This method has been applied to a variety of $2 \mathrm{D}$ free surface flows such as wave breaking on armor blocks (Altomare et al., 2014), regular and irregular waves propagation (Rota et al. (2014), Naeeja et al. (2017), Altomare et al. (2017), Roselli et al. (2018)) and wave overtopping (Suzuki et al., 2017). The Lagrangian formulation of the solver allows for multiple connected domains, with no need for a special treatment to solve for the free surface. This is exactly the characteristic that makes SPH models particularly appealing for studying violent freesurface flows (Violeau, 2012). Other SPH solvers have been used to simulate wave breaking phenomena (see e.g. Landrini et al. (2007) and Antuono et al. (2011)). Shao et al. (2006), Khayyer et al. (2008) and Liu et al. (2015) developed Incompressible SPH (ISPH) formulation and they applied it to the same category of problems. Shao et al. (2006) included turbulence effects into the spilling and plunging wave breaking prediction by coupling an incompressible SPH solver to the largely used $k-\epsilon$ turbulence model while Shao and Ji (2006) developed an SPH-LES model where turbulence of 2D plunging breakers has been described at particle and sub-particle scales.

The validation of the SPH model proposed in this paper is made against the experimental measurements on regular non-linear breaking waves performed by Kimmoun and Branger (2007). Despite the unique, high quality, phase averaged, flow characteristics of their experiments, the results have been rarely used to validate numerical simulation tools, in a similar long time simulation. The capability of the model to accurately predict 
the time-space evolution of the free surface, and the velocity fields is tested involving the whole experimental domain: propagation in deep water, shoaling in shallow water and breaking regions. Both singular wave characteristics of the train and the phase-average on 128 consecutive waves are considered. To the authors knowledge, it is the first time that numerical results are validated against experimental data on such a long evolution time though phase averaged field comparison. Makris et al. (2016) simulated 50 periods by using SPHysics to model weakly plunging breakers. They emphasize the importance of spatial discretization to properly capture the hydrodynamics in the surf region, but were limited by computation resources. The present study, based on DualSPHysics code, overcomes their computational limitations and at the same time, it proposes a detailed fluid dynamic comparison of the flow characteristics in the breaking and run up regions. Since most of the validation studies on periodic breaking waves compare results of one breaking event with the instantaneous experimental measurements, the present analysis addresses the repeatability and the accuracy of the SPH prediction on a larger number of waves, providing quantitative results of the detailed free surface flow field around periodic waves breaking and the run up on a sloped beach.

\section{Basic principles of wave breaking and run-up}

Several parametric formulations have been proposed attempting to predict the wave run-up on smooth- impermeable slopes. Hunt (1959) proposed an empirical formula based on laboratory experiments performed on either smooth or rough slopes and composite slopes. Considering the case of waves breaking on a slope, the non-homogeneous Eq. (1) for maximum run-up R above the Still Water Level (SWL) with respect to the wave height $\mathrm{H}$ reads as follows:

$$
\frac{R}{H}=\xi
$$

The constant $\xi$ is known as the Iribarren Number (Iribarren, 1949) or as surf-similarity parameter (Battjes, 1975), given as:

$$
\xi=\frac{\tan (\beta)}{\sqrt{\frac{H}{L_{0}}}}
$$

being $H$ the wave height, $\beta$ the slope angle and $L_{0}$ the wavelength in deep water given by the dispersion relation $L_{0}=\left(\frac{g T^{2}}{2 \pi}\right)$, where $T$ is the wave period. Several Authors (e.g. LLosada and Gimenez-Curto (1980),
Table 1: Iribarren Number for breaking waves

\begin{tabular}{cc} 
Type of breakers & $\xi$ \\
\hline Spilling & $<0.4$ \\
Plunging & $(0.4 ; 2.0)$ \\
Surging & $>0.2$
\end{tabular}

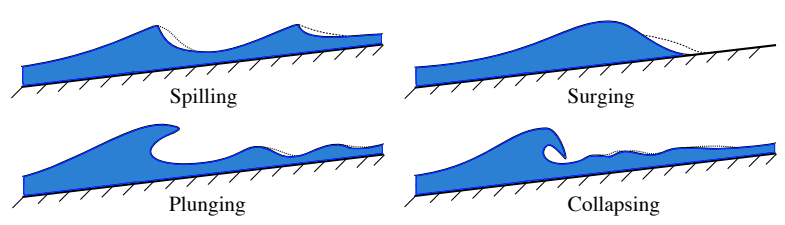

Figure 1: Schematic representation of the types of wave breaking according to (Galvin Jr, 1968)

Hughes (2004), and Hsu et al. (2012)) confirm that Eq. (1) provides suitable results for $\xi<2.5$ even if accurate predictions of the run-up are missed for $\xi>2.0$. $\xi$ is usually computed by using a finite-depth local wave height in the proximity of the slope toe rather than the true deep-water $H_{0}$. According to Table 1, depending on $\xi$, value wave breaking is classified in different types, namely spilling, plunging and surging as sketched in Figure 1 (Galvin Jr, 1968). Attempting to predict runup for breaking and non-breaking waves, Schuttrumpf (2001) proposed the following hyperbolic formula:

$$
\frac{R}{H}=a \cdot \tanh (b \cdot \xi)
$$

where the empirical coefficients are taken as $a=2.25$ and $b=0.5$, respectively. Even if several studies have providing criteria to predict the onset of wave breaking (Nepf et al. (1998), Wu and Nepf (2002), Oh et al. (2005) and Tian et al. (2008)), none of them has been universally accepted in practice. Geometric-based breaking formulations describe the limiting wave height in terms of the maximum wave steepness or the breaker depth index. Chue (1980) proposed a single equation (see Eq. (4)) for breaking and non-breaking waves by unifying previous theoretical and experimental formulae:

$$
\frac{R}{H}=1.8\left(1-3.1 \frac{H}{L_{0}}\right) \xi_{0}\left(1-\exp \left(\frac{\pi}{2 \beta}\right)^{\frac{1}{2}} \frac{1}{\xi_{0}}\right)
$$

being:

$$
\xi_{0}=\frac{\tan (\beta)}{\left(\frac{H}{L_{0}}\right)^{0.4}}
$$

Ahrens and Titus (1985) however demonstrate that the Eq. (4) is not universally applicable. Hughes (2004) 
presented a new method for predicting the run-up from non-breaking waves, proposing the run-up formula of Eq. (6) based on a momentum flux parameter rather than on $\xi$.

$$
\frac{R}{H}=3.84 \tanh \left(\frac{M_{f}}{\rho g h^{2}}\right)^{\frac{1}{2}}
$$

The ratio $\frac{M_{f}}{\rho g h^{2}}$ represents the maximum depth-integrated wave momentum flux before reaching the breaking event. This formula fails to predict the run- up on the steeper slopes $\left(\beta>30^{\circ}\right)$. Eq. (6) states that the relative run-up $R / H$ is a function of the slope by the term $\tan (\beta)$. This means that the run-up increases with the slope of the structure. This has been confirmed by the observations carried out by Hsu et al. (2012) who provide the following Eq. (7):

$$
\frac{R}{H}=2(0.5 \xi)^{\frac{0.04}{\tan ^{2}(\beta)}}
$$

\section{Reference experimental tests}

As mentioned the experimental measurements from Kimmoun and Branger (2007) have been selected for the validation of the used SPH solver. The very comprehensive set of fluid dynamic data measured in this study offers the unique opportunity of detailed valuation of the proposed SPH solver ability to reproduced breaking wave. A side view of the tank used for the experiment at ECM/IRPHE with the test set-up is shown in Figure 2. The tank is $17 \mathrm{~m}$ long and $0.65 \mathrm{~m}$ widths. The water depth in front of the wavemaker is $0.705 \mathrm{~m}$. The beach slope is $1 / 15$ with smooth polyvinyl bottom. The sloped beach is about $13 \mathrm{~m}$ long. The wave reflection coefficient considering the flat part of the wave tank is less than $2 \%$. The sloped bottom starts after $4 \mathrm{~m}$ from the wavemaker. Wave elevation has been measured with a set of six evenly spaced resistive wave gauges, with a distance of $18 \mathrm{~cm}$ from each other. The velocity field in the breaking region has been measured by means of Particle Image Velocimetry (PIV) technique on 14 different windows distributed over the surf zone. The detection zone begins $3 \mathrm{~m}$ before the intersection between the beach and the still water level. The frequency of acquisition of the breaking phenomena is $200 \mathrm{~Hz}$. The single paddle wavemaker generates regular waves by sinusoidal motion. The wave period is $\mathrm{T}=1.275 \mathrm{sec}$, and the observed wave amplitude before the sloped beach is $a=5.70 \mathrm{~cm}$. The wavelength at $x=4.00 \mathrm{~m}$ (the wavemaker is located at $\mathrm{x}=0 \mathrm{~m}$ ) is $\lambda=2.41 \mathrm{~m}$ and the wave height at breaking is $H_{B}=14.00 \mathrm{~cm}$. For each run, 128 wave-cycles have been waited before start acquiring the data. Image acquisition lasts for $163.2 \mathrm{sec}$, corresponding to 128 wave periods.

\section{Smoothed Particle Hydrodynamics Method}

The following Section 3.1 and 3.2 give the theoretical background of the SPH solver used in the study and the set-up of the numerical simulation.

\subsection{DualSPHysics formulation backgrounds}

DualSPHysics is a weakly compressible Smoothed Particle Hydrodynamic (SPH) open source solver see Gomez-Gesteira et al. (2012) and Crespo et al. (2015) under continuous implement and recently used for different coastal hydraulic studies Altomare et al. (2014, 2017). SPH is a mesh-free Navier-Stokes equation solver where the fluid is represented by a discrete number of particles whose kinematics and dynamics are solved. Physics variables such as position, velocity and pressure are computed at any point in the fluid by interpolation of the flow characteristics carried by each particle within a support domain. SPH method develops in two major phases, namely a kernel and a particle approximation. Within the Kernel approximation, a function $F(r)$ and its derivatives are substituted in a continuous by an integral form with a suitable kernel function $W$ as in Eq. (8). Such a kernel function $W$ must fulfill several properties (Monaghan, 1992). Its support domain is defined by the so-called smoothing length $h$. Eq. (8) is then further approximated at each particle. Considering a particle $a$, the contributions from all neighbor particles within the support domain of the

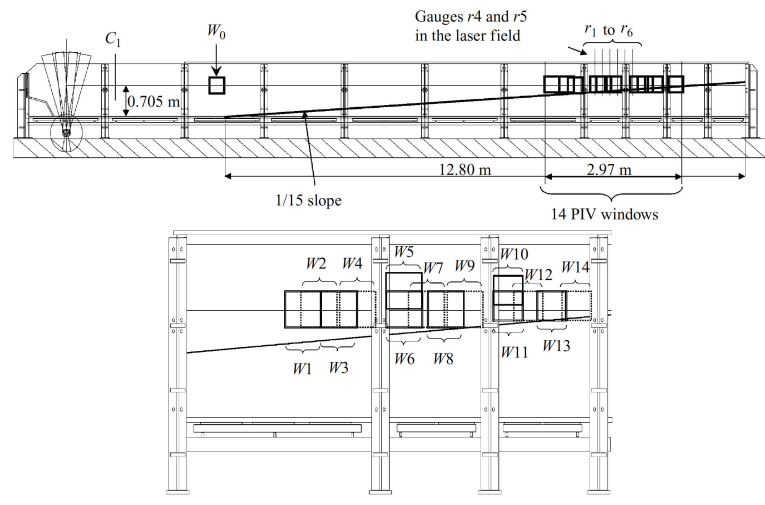

Figure 2: Side view and set-up of the wave flume used in the experimental campaign (Kimmoun and Branger, 2007). 
kernel are summed as in Eq. (9).

$$
\begin{gathered}
F(r)=\int_{\Omega} F\left(r^{\prime}\right) W\left(r-r^{\prime}, h\right) d r^{\prime} \\
F\left(r_{a}\right) \approx \sum_{b} F\left(r_{b}\right) W\left(r_{a}-r_{b}, h\right) \frac{m_{b}}{\rho_{b}}
\end{gathered}
$$

Among the kernel functions available in DualSPHysics, the Cubic kernel proposed by Monaghan and Lattanzio (1985) has been selected. This kernel resembles a Gaussian function but with a narrower compact support. It is defined according to Eq. (10):

$$
W(r, h)=\alpha_{D} \begin{cases}1-\frac{3}{2} q^{2}+\frac{3}{4} q^{3} & 0 \leq q \leq 1 \\ \frac{1}{4}(2-q)^{3} & 1 \leq q \leq 2 \\ 0 & q \geq 2\end{cases}
$$

Where $\alpha_{D}=\frac{10}{7 \pi h^{2}}$ in 2D and $\alpha_{D}=\frac{1}{\pi h^{3}}$ in 3D to ensure normalization requirement. A tensile correction Monaghan (2000) is automatically active when using kernels with first derivatives that go to zero with decreasing inter-particle spacing. Being $v$ the velocity, $P$ the pressure, $\rho$ the density, $m$ the mass, $g$ the gravitational acceleration, the particle acceleration is computed again considering the interactions within the support domain according to the Eq. (11) proposed by Monaghan (1992):

$$
\frac{d \mathbf{v}_{a}}{d t}=-\sum_{b} m_{b}\left(\frac{P_{b}^{2}}{\rho_{b}}+\frac{P_{a}^{2}}{\rho_{a}}+\Pi_{a b}\right) \Delta_{a} W_{a b}+\mathbf{g}
$$

Where $\Pi_{a b}$ is the artificial viscosity term given in Eq. (12):

$$
\Pi= \begin{cases}\frac{\alpha \mu_{a b} \bar{c}_{a b}}{\rho_{a b}} & \mathbf{v}_{a b} \mathbf{r}_{a b}<0 \\ 0 & \mathbf{v}_{a b} \mathbf{r}_{a b}>0\end{cases}
$$

where $\mathbf{r}_{a b}=\mathbf{r}_{a}-\mathbf{r}_{b}$ and $\mathbf{v}_{a b}=\mathbf{v}_{a}-\mathbf{v}_{b}$ and $\mathbf{r}_{k}$ and $\mathbf{v}_{k}$ are the particle position and velocity, respectively, $\mu_{a b}=$ $\frac{h \mathbf{v}_{a b} \cdot \mathbf{r}_{a b}}{r_{a b}^{2}+\eta^{2}}$ and $\eta^{2}=0.001 h^{2}$. The mean speed of sound is $\bar{c}_{a b}=0.5\left(c_{a}+c_{b}\right)$ and $\alpha$ is a viscosity coefficient used to tune the dissipation in the numerical simulation. A reference value is $\alpha=0.01$ (Altomare et al., 2015). Changes in fluid density are computed by solving the conservation of mass, which is constant for all particles. In SPH form it is written as in Eq. (13):

$$
\frac{d \rho_{a}}{d t}=\sum_{b} m_{b} \mathbf{v}_{a b} \cdot \Delta_{a} W_{a b}
$$

Pressure is computed from density values of the particle by using a stiff equation of state, as in Eq. (14) (Batchelor, 2000):

$$
P=B\left[\left(\frac{\rho}{\rho_{0}}\right)^{\gamma}-1\right]
$$

being $B=\frac{c_{0}^{2} \rho_{0}}{\gamma}, \gamma=7$. The compressibility is tuned to lower the speed of sound in order to keep a reasonable value of the computational time step. The latter is in fact computed according to a Courant-Friedrichs-Lewy (CFL) condition based on the actual calculated speed of sound for all particles. The speed of sound $c_{0}$ is defined at the reference density as in Eq. (15):

$$
c_{0}=\sqrt{\left.\frac{\partial P}{\partial \rho}\right|_{\rho_{0}}}
$$

The speed of sound is computed to maintain the Mach Number, $M a=\frac{v}{c_{0}}$, in the subsonic weakly-compressible regime to avoid the propagation of fast sound waves. The latter is taken as the wave- front velocity of a dambreak:

$$
c_{0}=\operatorname{coef}_{\text {sound }} \sqrt{g h_{\text {swl }}}
$$

$h_{s w l}$ is the still water level and coe $f_{\text {soud }}$ is a coefficient in the range [10-30]. The Symplectic algorithm Leimkuhler and Patrick (1996) has been used for time integration. It is an explicit second-order scheme with accuracy in time of $O\left(\Delta t^{2}\right)$ involves a predictor and a corrector step. A variable time step $\Delta t$ is computed at each iteration according to Monaghan and Kos (1999), as in Eq. (17), based on the CFL condition, the force terms and the viscous diffusion term:

$$
\left\{\begin{array}{l}
\Delta t_{f}=C F L \cdot \min \Delta t_{f}, \Delta t_{c v} \\
\Delta t_{f}=\min _{a} \sqrt{\frac{h}{\left|f_{a}\right|}} \\
\Delta t_{c v}=\min _{a} \frac{h}{c_{s}+\max _{b} \mid \frac{h_{a b} \cdot \mathbf{r}_{a b}}{\left(r_{a b}^{2} \eta^{2}\right)}}
\end{array}\right.
$$

$\Delta t_{f}$ is based on the force per unit mass $\left|f_{a}\right|$, and $\Delta t_{c v}$ includes both the Courant and the viscous time step controls. Dynamic boundary condition is used in DualSPHysics (Crespo et al., 2007). According to this approach, the boundary particles satisfy the same continuity equation as the fluid particles, and their density and pressure also evolve. In this way the density of the boundary particles increases, giving rise to an increased pressure value; the force exerted on the fluid particle also increases because the pressure term in the momentum equation creates a repulsive mechanism between the fluid and boundary particles. However they do not move according to the forces exerted on them. According to this formulation of the boundary condition, as a fluid particle approaches a boundary particle beyond the kernel range, the density of the boundary particles increases. This generates an increase of the pressure resulting in the repulsive force. Wave generation is achieved using a moving boundary that mimics 
the displacement of a wavemaker in experimental wave tank.

\subsection{SPH model setup}

The tank geometry used in the experiment has been exactly reproduced in the SPH model including the wave maker and the measurement area as shown in Figure 3. Table 2 reports the set of the main SPH parameters used in the simulations. The choice of these parameters is often not obvious and it has been made on the basis of previous systematic studies about the effect of numerical and physical parameters of SPH models for simulation of free surface waves (Roselli et al., 2018). Each of the selected parameters has been changed within specific bounds imposed either by physical considerations (e.g. on coef $f_{\text {sound }}$ (Meringolo et al., 2017)) or by numerical stability constraints (e.g. on the $C F L$ number (Violeau and Leroy, 2015)). In this study, the criteria used for optimal parameter selection has been the minimum error on the simulated free surface elevation of the non-linear periodic waves propagating in deeper water, in the region upstream the sloped beach. The accuracy level is evident in the comparison of Figure 4. The error on wave elevation is less than $3 \%$ (RMS) with respect to the experimental waves. This choice does not in principle ensures that the same combination of parameters will be the most suitable for breaking events too. However, since hundreds of waves need to be reproduced the priority has been given to this stage, verifying a-posteriori the performance at the surf zone.

The numerical tank has been filled with about $3 M L$ particles in order to ensure an adequate resolution at the surf zone. In particular wave height has been resolved by using 63 particles, according to the ratio $\frac{H}{d p}=63.3$. The coefficient coe $f_{h}=1.8$ leads to a smoothing length $h=\operatorname{coef}_{h} \sqrt{2 d p^{2}}=4.58 \cdot 10^{-03}[\mathrm{~m}]$. Simulations have been run on the HPC cluster at CINECA Supercomputing Center by using GP-GPU Nvidia K40, resulting in about 240 hours of effective computational time required for 350 seconds of physical simulation time. The output time for numerical data acquisition (different from the variable time step discussed in the previous section) has been set to $\Delta t_{\text {out }}=0.05 \mathrm{sec}$. The free surface and the fluid particle velocity field is then sampled at the corresponding frequency $(20 \mathrm{~Hz})$. The free-surface elevation is computed based on the interpolated mass, i.e. if the particle mass is higher than a reference mass value its height will be considered as the free surface elevation. The velocity field has been computed over a (discrete) rectangular grid of points refined

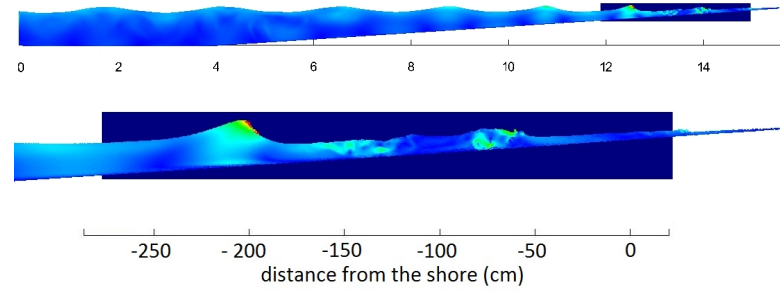

Figure 3: Snapshot of the SPH simulation where the whole computational domain is visible. A zoom close to the measurement area over the surf zone is shown too. Color-map represents fluid velocity.

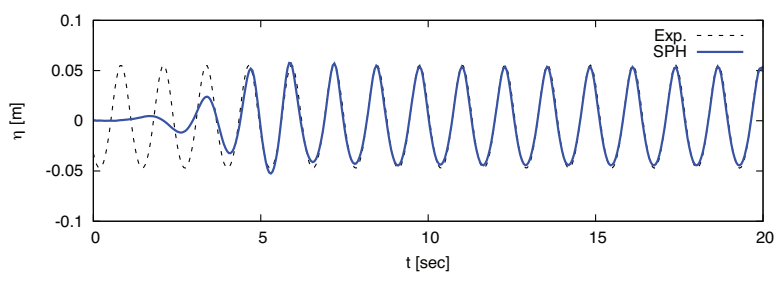

Figure 4: Comparison between the experimental (black dotted) and simulated (blue solid) wave elevation versus time measured at the toe of the beach $(\mathrm{x}=4 \mathrm{~m})$.

over the surf zone, i.e. in correspondence to the sloped beach.

The measurement grid starts at $x=11.85 \mathrm{~m}$ from the wavemaker and develops longitudinally for a length $L_{\text {grid }}=3 \mathrm{~m}$. In vertical direction it develops for a height $h_{\text {grid }}=0.30 \mathrm{~m}$. Uniform spacing between the points have been used both in longitudinal and vertical directions, with $\Delta x=0.592 \mathrm{~cm}$ and $\Delta z=0.094 \mathrm{~cm}$, respectively. This numerical grid exactly mimics the one used to get the experimental measurements.

\section{Results and discussion}

The comparison between experimental and numerical results is performed in terms of instantaneous, mean and phase averaged wave characteristics. Figure 4 displays the wave elevation over 20 seconds at the toe of

Table 2: SPH model parameters.

\begin{tabular}{lc} 
Parameter & Value \\
\hline $\mathrm{dp}$ & $1.80 \cdot 10^{-03}[\mathrm{~m}]$ \\
CFL & 0.176 \\
coef $_{h}$ & 1.80 \\
coef $_{\text {sound }}$ & 15.8 \\
$\alpha$ & 0.01
\end{tabular}



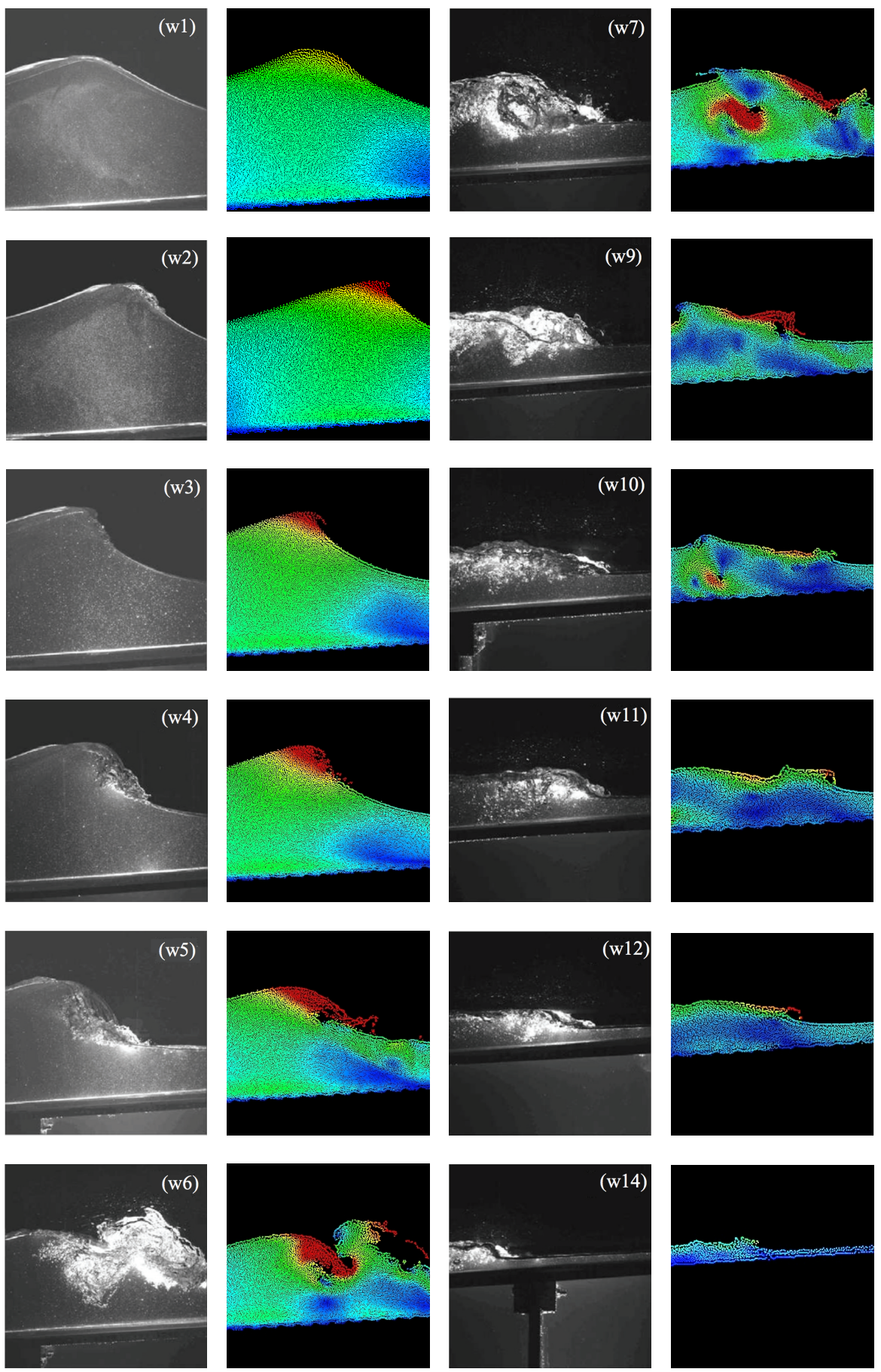

Vel Magnitude

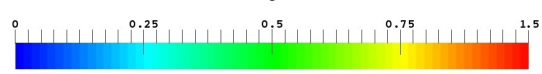

Figure 6: Comparison of the numerical and experimental wave evolution. Same windows used in the experiments are used for the numerical simulations (second and fourth columns). Velocity is expressed in $[\mathrm{cm} / \mathrm{sec}]$. 

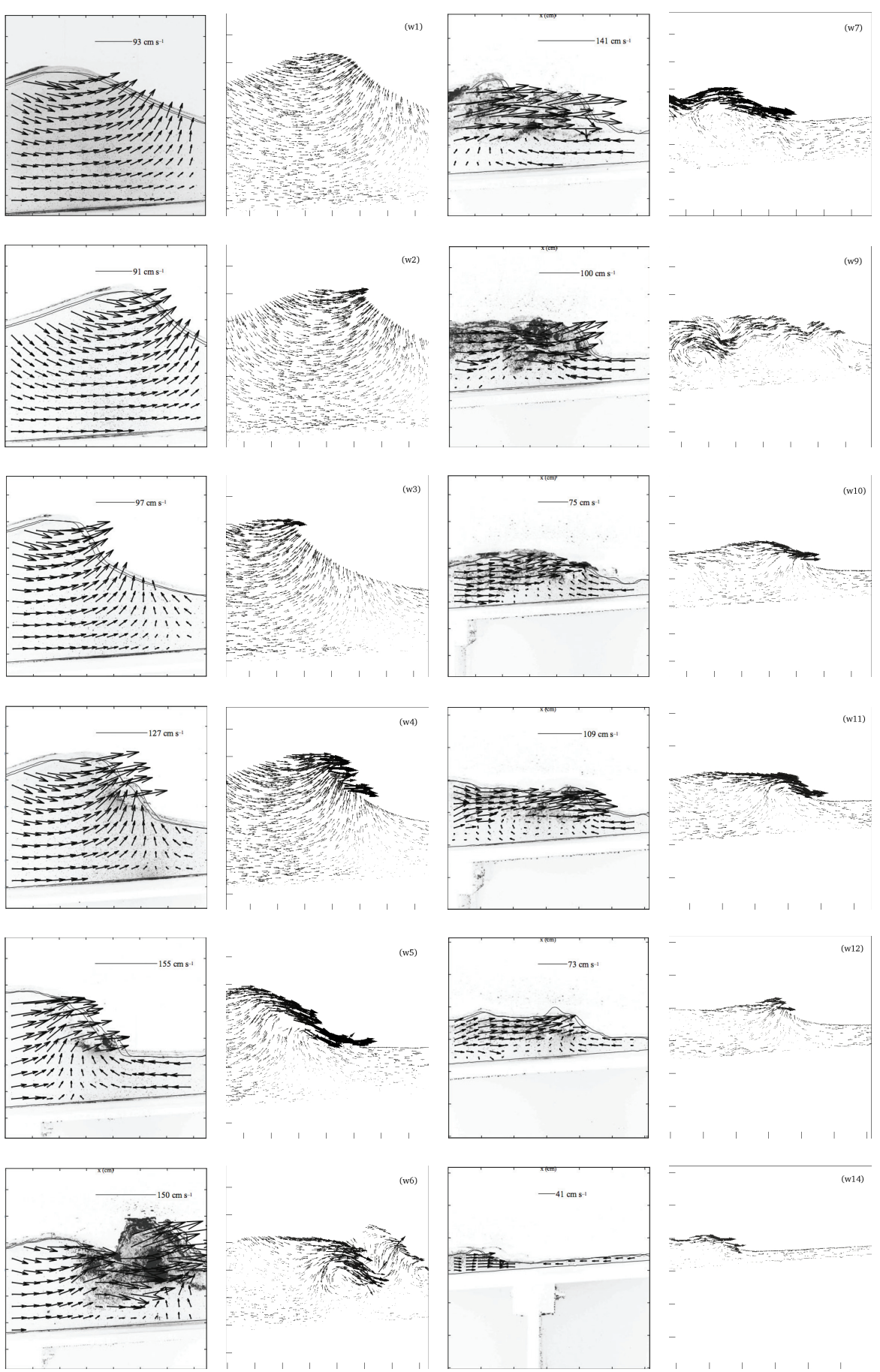

Velocity X

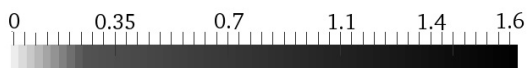

Figure 7: Instantaneous experimental and numerical velocity field evolution, represented with arrow vector (grey intensity lengths are proportional to the velocity component in the $\mathrm{x}$ direction). Experimental results are shown on the first and third columns. SPH results are shown on the second and fourth column. Velocity is expressed in [cm/sec]. 
the sloped beach located at $x=4.0 m$ from the wavemaker. The still water level corresponds to $z=0.0 \mathrm{~m}$. The numerical wave elevation is in good agreement with the experimental measurement. This first verification ensures that the waves that reach the surf zone are comparable to those from the experiments. The prediction of the instantaneous free surface elevation at three locations over the breaking zone is shown in Figure 5 for 8 subsequent wave periods. The three locations, i.e. $x=$ $248 \mathrm{~cm}, x=142 \mathrm{~cm}$ and $x=42 \mathrm{~cm}$, respectively, are representative of three distinct events: incipient breaking, wave plunging with the consequent splash-up and the wave roller, respectively. The agreement is again very satisfactory especially in the first phases of the wave breaking, i.e. the incipient breaking. The correspondence between numerical predictions and experimental measurements slightly degrades during the breaking, splash-up and roller phases. The maximum errors on the crests $\epsilon_{c}$ and the troughs $\epsilon_{t}$ are equal to $\epsilon_{c}=4 \%$ and $\epsilon_{t}=7 \%$ during the splash-up and to $\epsilon_{c}=8 \%$ and $\epsilon_{t}=12 \%$ during the roller phase. This can be explained by the presence of large air entrainments in the experimental wave breaking events that are not properly resolved in this SPH model. This is clearer, analyzing Figure 6 that shows the comparison of the snapshots of a wave passing through some of the fourteen windows of the tank. Incipient breaking starts at windows \#3, immediately followed by the plunging with an overturning lip and splash-up in windows \#4 and \#5, respectively. Then wave roller phase starts at windows \#6. The waves broke initially at about $x=250 \mathrm{~cm}$ from the shoreline. The wedge of water pushed up by the plunging jet forms another splash-up jet which strikes the water ahead of it at a second plunging point as rapresented in the window \#7. From the window \#8 to \#11 the wavefront moves into a roller propagating towards the shore. The wave crosses the shoreline in the window \#14 and arrive up to the maximum run-up point. After this point the runs up flow start to coming back. The foam that begins to develop with the plunging wave increases as the wave breaks and it persists at least until windows \#9. Even if the SPH resolves a single fluid phase, namely the water phase, the global wave breaking dynamics is reasonably captured due to the correct modeling of the particle interactions. Marrone et al. (2016) used a multi-phase SPH solver for a shallow water breaking wave. They found a "quite different flow evolution after the plunging breaker touches the interface" while no relevant differences in terms of global phenomenon, measured by the dissipated energy, have been achieved. Compressibility effects due to the presence of an air phase are here neglected but the density change of the foamy phase is
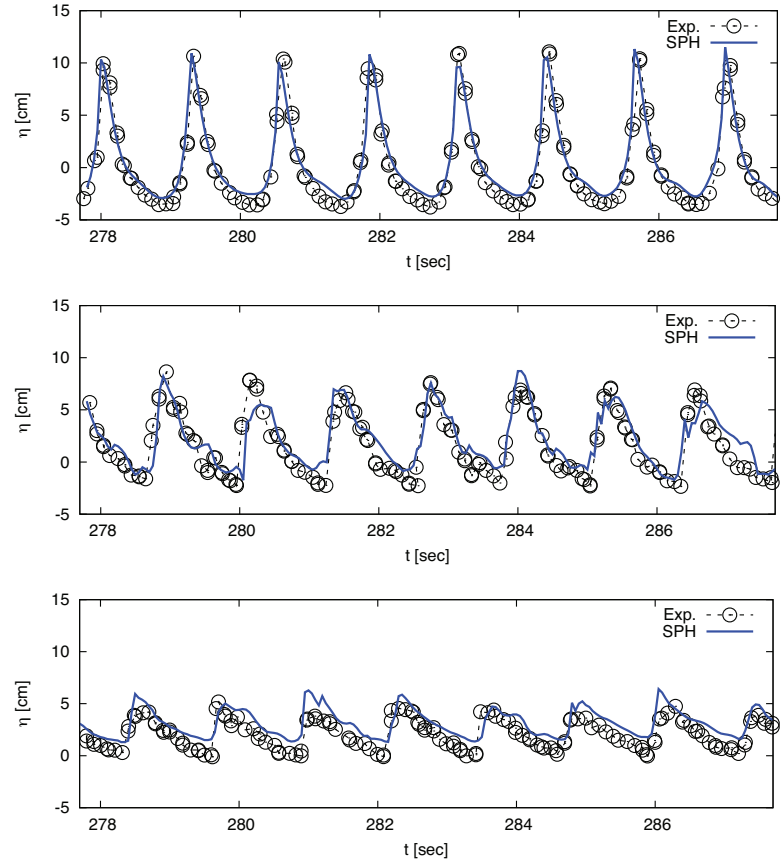

Figure 5: Comparison between the experimental (black circles) and simulated (blue solid) time history of the wave elevation at three different distances from shoreline: (a) $248 \mathrm{~cm}$, (b) $142 \mathrm{~cm}$ and (c) 42 $\mathrm{cm}$.

captured by the particle rarefaction. This appears e.g. in window \#6 of the numerical solution shown in Figure 6. The same comparison but in terms of velocity vector field is shown in in Figure 7. Water flow over the same windows is displayed. The directions of the velocity vectors are well captured up to incipient breaking and during the rolling phase. Major differences are found when the plunging wave collapses, splashing-up into the foremost water (see window \#6). Compared to the experimental reconstructions, the magnitude of the fluid particle speed predicted by the SPH during the rolling phase appears to be slightly under-estimated at the free surface (windows \#7 and \#9). This might be related to both the smoothing length and the artificial viscosity used in the numerical model. The first is in fact responsible of the interactions among neighbor particles while the latter reproduced damping effects due to the viscosity. Their combination might strongly affect the both the kinematics and dynamics of the free surface (Roselli et al., 2018). Figure 8 provides a global picture of the above-discussed instantaneous wave characteristic. It shows the envelop of the max and minimum wave elevations at any point over the whole simulation time. The mean water level is simply deduced as the average of the maximum and the minimum envelope. The value 


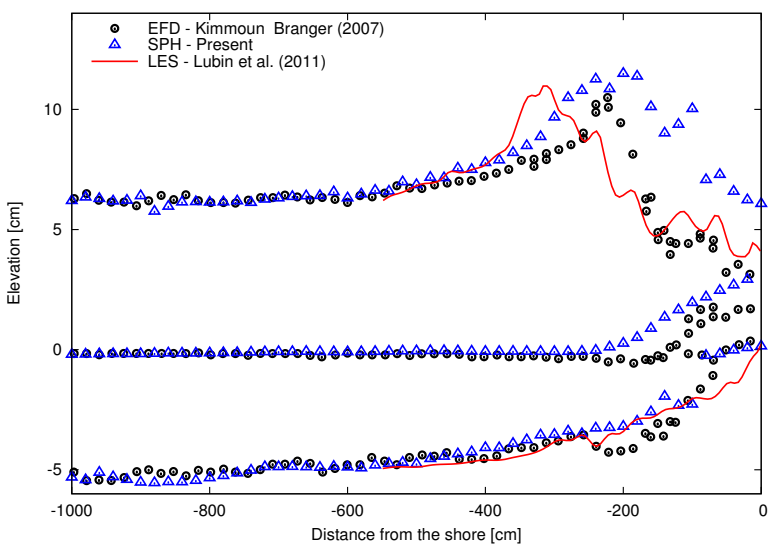

Figure 8: Comparison between the experimental (black circles) and simulated (blue triangles) envelops of the minimum (troughs), mean and maximum (crests) water levels. Results from LES computations REF are shown by red curves.

of the wave height at breaking (max value of the max envelope) is correctly predicted but the maxima envelope curve is less peaked than the experiments. Close to wave breaking, the error in term of maximum water level is about $\epsilon_{c}=12 \%$. The minimum and the mean water level are more closely reproduced also after the breaking. It is important to notice that there is a difference in the method used to measure the free surface elevation in surf zone between the experimental and the numerical measurements. The instantaneous free surface level of the SPH simulation is obtained by measuring the level of the highest particle at the given longitudinal location, no smoothing has been used. The experimental technique to measure the free surface elevation at a section of the channel is surely affected by a certain spatial and time averaging (or smoothing) in presence of a foamy mixture of air and water as one can expect in the surf region. In Figure 8 results from Large Eddy Simulations (LES) by Lubin et al. (2011) are shown too. This latter set of results anticipate the incipient breaking while shows a better prediction of the mean crests after this event. This can be exactly related to the multi-phase solution provided by the LES computations. The probability density function of the maximum run up point over the whole set of simulated waves is shown in Figure 9. The run up is here presented as percentage deviation from the still water level and it refers to a vertical elevation. A best-fit curve is shown too. The mean value obtained by this discrete distribution is $\frac{R}{H} \cong 7.53 \%$ while its standard deviation is $\sigma \frac{R}{H} \cong 1.03 \%$. Table 3 gives the comparison between

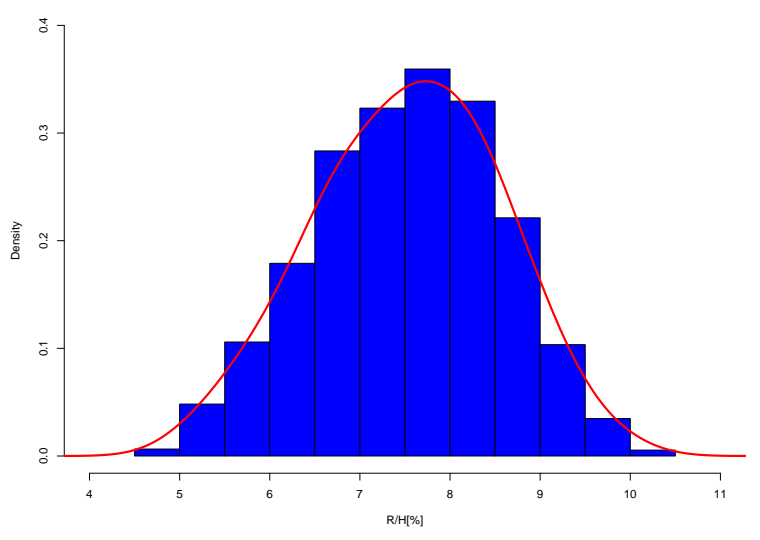

Figure 9: Run up distribution expressed as percentage deviation from still water level over the entire simulation time. Fit curve is shown in red.

Table 3: Run up error between SPH results and empirical formulations.

\begin{tabular}{cc} 
Method & Run Up \\
\hline SPH & $7.53 \%$ \\
Eq. (1) & $15.62 \%$ \\
Eq. (3) & $13.78 \%$ \\
Eq. (4) & $9.87 \%$ \\
Eq. (6) & $12.34 \%$
\end{tabular}

numerical results and approximate formulations of Eq. (1), Eq. (3), Eq. (4) and Eq. (6). The following values have been used for the purposes of this comparison: $\xi=0.31, \beta=0.067 \mathrm{rad}, H=0.11 \mathrm{~m}$ and $L_{0}=2.54 \mathrm{~m}$. It appears that SPH run-up prediction is slightly below all the approximate solutions. The numerical solution of the run-up is, on average, in better agreement with one of the most recent criterion, i.e. the empirical formula of Eq. (3) proposed by Schuttrumpf (2001). Eq. (4) over-predicts the wave amplitude at breaking by a factor two with respect to the SPH result.

As anticipated, a goal of this study is the assessment of the repeatability of the simulated results over multiple (in excess of one hundred) periodic events. To this aim, phased averaged wave characteristics have been analyzed both in terms of free surface $\eta_{\phi_{i}}(x)$ and fluid velocity $v_{\phi_{i}}(x, z)$. According to Kimmoun and Branger (2007) such phase averages have been computed as follows:

$$
\left\{\begin{array}{l}
\eta_{\phi_{i}}(x)=\frac{1}{n_{w}} \sum_{j=1}^{n_{w}} \eta\left(x, t_{i, j}\right) \\
v_{\phi_{i}}(x, z)=\frac{1}{n_{w}} \sum_{j=1}^{n_{w}} v\left(x, z, t_{i, j}\right)
\end{array}\right.
$$

where the time instant $t_{i, j}$ is chosen so that waves 

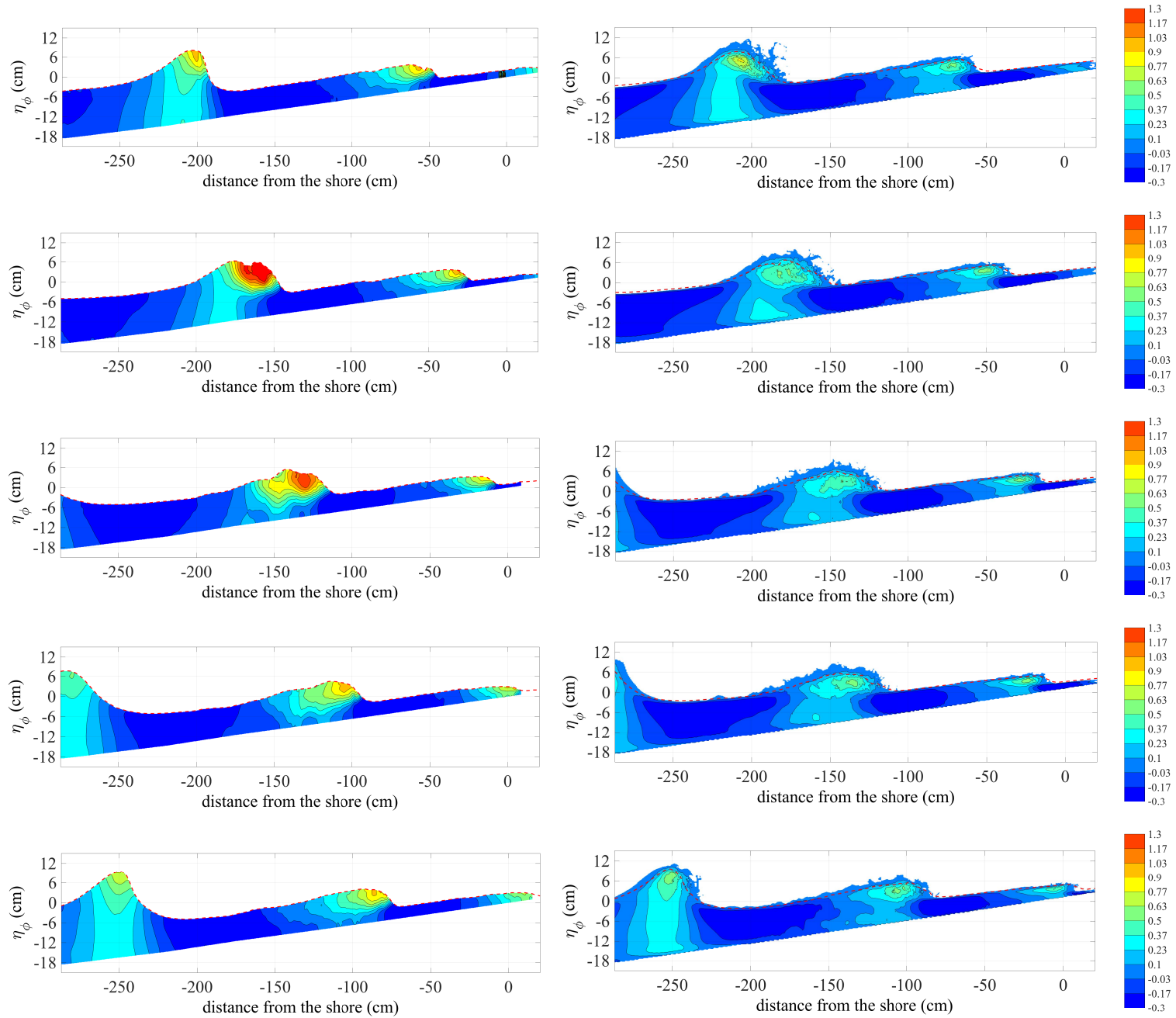

Figure 11: Comparison of the experimental (left column) and computed (right column) horizontal component of non-dimensional phase-averaged velocities for the phases $\phi=\left[35^{\circ}, 106^{\circ}, 176^{\circ}, 247^{\circ}, 318^{\circ}\right]$. Each row corresponds to a given wave phase. Velocity is expressed in non-dimensional form as $U_{\phi}(x, z) / \sqrt{g D(x)}$.

with the same phase $\phi_{i}$, within a wave period $T$, are averaged. The phase average is performed on the last 128 following waves $\left(n_{w}=128\right)$ of the numerical simulation. Figure 10 shows examples of numerical versus experimental free surface phase-to-phase repeatability. The results on the phase averaged free surface are consistent with the instantaneous values shown in Figure 6 and the max and mean wave characteristics shown in Figure 7, respectively. The maximum wave height is well predicted both in terms of location over the sloped beach and in terms of absolute value. This means that the water kinematics at incipient breaking up to the beginning of plunging is captured in a stable and repeatable way by the numerical model. After wave breaking, closer to the shore, the height of the free surface is, on average, over estimated due to the splash-up events, but its general shape continues to be correctly computed by the SPH. The maximum deviation from the experiments is less than $2 \mathrm{~cm}$. The same offset seems to be maintained up to the shoreline. The horizontal component of the non-dimensional phase averaged velocity $v_{\phi_{i}}(x, z)$ is compared in Figure 11 for five different phases, namely $\phi=\left[35^{\circ}, 106^{\circ}, 176^{\circ}, 247^{\circ}, 318^{\circ}\right]$. This set of phases allows spanning the whole wave period $[0,2 \pi]$. To the author's knowledge it is the first time that the comparison between numerical and experimental phase-average results is presented in the space-domain (e.g. $\left.U_{\phi}(x, z)\right)$ over the surf zone; previous published results have usually presented comparison in the time-domain (e.g. 

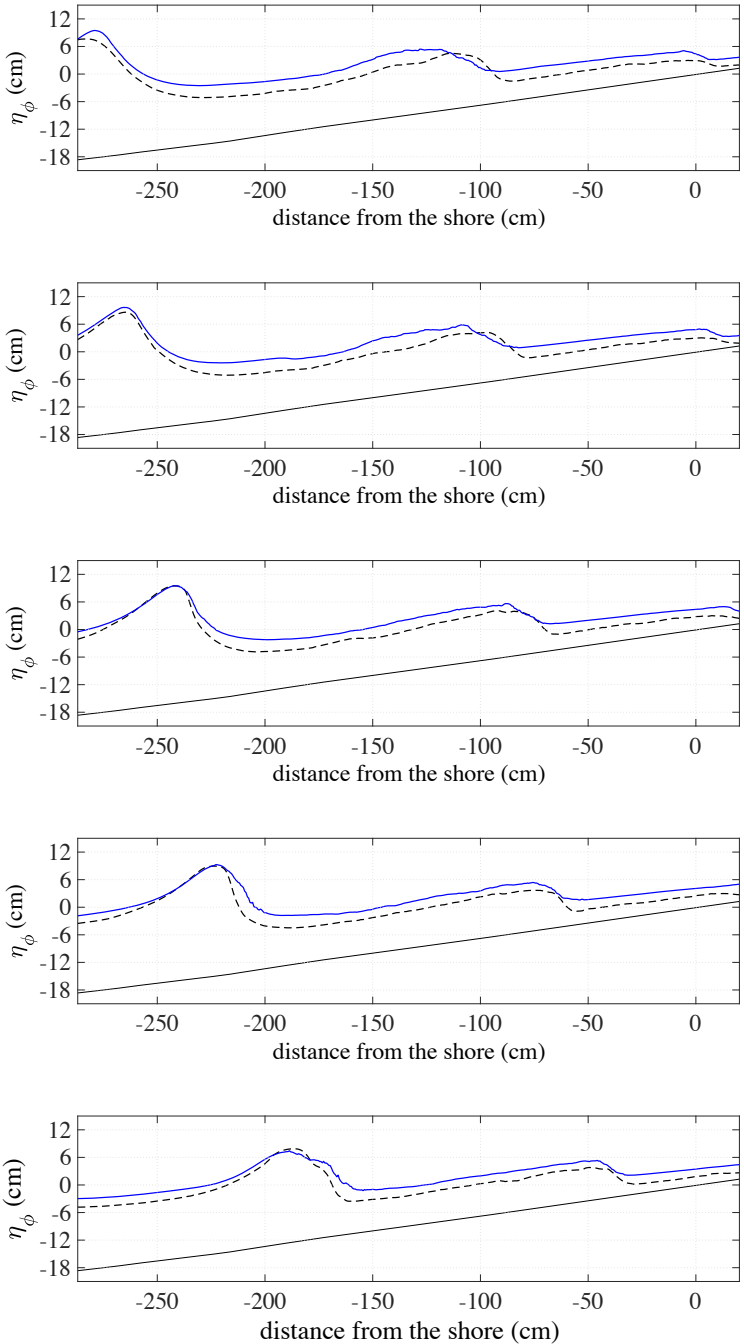

Figure 10: Phase averaged of free surface elevation over 128 subsequent wave cycles. Blue solid lines and black dashed lines represent SPH and experimental results, respectively, at phases $\phi=$ $\left[35^{\circ}, 106^{\circ}, 176^{\circ}, 247^{\circ}, 318^{\circ}\right]$ from top to the bottom.
$U_{\phi}(t)$ at each measurement point) for only one wave cycle.

In the experimental data by Kimmoun and Branger (2007) a cubic smoothing spline interpolation has been used to construct the velocity field over the whole surf zone instead the superposition of numerical results has no smoothing and is showed without any interpolations. Since the numerical model captures all the sprays of the free surface, the latter appears more irregular in the numerical simulations compared to that of the experimental tests. Results clearly show the wave height attenuation during the wave breaking, and the decreasing of the wavelength as the wave reaches the shoreline. Velocities are positive (shoreward) in correspondence of the wave crests, over the whole water column from the top of the crest to the bottom. Similarly, velocities are negative (seaward) in correspondence of the troughs. The first order approximation of the wave celerity $\sqrt{g D(x)}$ at water depth $D(x)$ is considered for the comparison. The maximum velocity is underestimated by the SPH model of about $12.25 \%$, being $c(x)_{E X P}=1.20 \sqrt{g D(x)}$ and $c(x)_{S P H}=1.05 \sqrt{g D(x)}$ at the top of the crests. Just after the splash-up zone the error rises at $18.51 \%$, being the experimental and numerical celerity $c(x)_{E X P}=1.35 \sqrt{g D(x)}$ and $c(x)_{S P H}=1.10 \sqrt{g D(x)}$, respectively. Negative velocities are equal to $-0.40 \sqrt{g D(x)}$ under the troughs. The numerical results show that the distribution of the horizontal velocity is correctly reproduced during the wave evolution.

The Dynamic Boundary Condition (DBC) implemented in DualSPHysics (Crespo et al., 2007) explains the differences of the phase averaged velocity fields close to the bottom. In fact the boundary layer is usually reproduced by means of a no-slip boundary condition by which the tangential velocity at the wall is nullified. By using the DBC the boundaries are treated as solid impermeable walls, meaning that the boundary particles will exert a repulsive force on the fluid particles as their relative distance becomes smaller than twice the smoothing length.

The highest velocities occur at the same vertical line under the wave crest where the water column are all located at $x=-240 \mathrm{~cm}$ (e.g. at $\phi=318^{\circ}$ ). After the maximum velocity position, there is a phase shift from the bottom to the wave crest. Velocities are in advance of phase compared to bottom velocities. This phase shift is visible especially at $\phi=318^{\circ}$ both in the experiments and in the SPH. 


\section{Conclusions}

An extended validation study of the capabilities of an optimal SPH model for the simulation of wave transformations in the surf zone has been presented over a large number (128) of consecutive events. Numerical predictions of non-linear regular wave breaking over a sloped smooth beach have been compared to high-quality experimental flow field measurements performed in a wave flume. Wave characteristics are studied in terms of instantaneous wave profiles and fluid velocities, mean free surface elevation and run-up prediction and phase-average free surface and fluid velocities, respectively. The quantitative accuracy of the SPH model on the instantaneous wave profiles has been defined at three distances from the shoreline corresponding to incipient breaking, plunging (and splash-up) and wave roller, respectively. The maximum errors on the wave crests and troughs are lower than 7\% at plunging event and lower than $12 \%$ during the roller phase. The simulation quality of the wave breaking kinematics has been also qualitatively confirmed by a series of instantaneous flow snapshots over the 12 inspection windows used in the experiments. The mean location of the wave breaking is well predicted as well as the minimum and the mean water level. The mean run-up obtained by the SPH simulation, equal to $\frac{H}{R} \cong 7.53 \%$, has been compared to approximate solutions from literature since this data has not been measured during the experimental campaign. This comparison reveals that the differences between the SPH average prediction on all the wave periods and the minimum and the maximum run up empirical values are equal to $4.33 \%$ and $6.25 \%$, respectively. Considering the uncertainties related to the approximate formulations these results are quite satisfactory. The phase-to-phase repeatability of the free surface elevation confirms the reliable prediction of the averaged wave profile up to breaking event. A relatively small error, in the order of $10 \%$, is found after breaking where the free surface is extremely non-linear and foam inclusions, which are not modeled in the SPH solver, are significant. The present model appears to adequately predict the distribution of the phase-averaged horizontal component of the velocity in the surf zone. However larger errors are found on the velocity parameter. In particular, with reference to the first order approximation of the wave celerity $\sqrt{g D(x)}$, the maximum velocity under the crest is under predicted of $12.25 \%$ and after splashup this difference rises up to $18.51 \%$. Overall the phase averaged results obtained from the SPH model having optimal parameters, seem to provide satisfactory indications about the wave transformation in the surf-zone and run-up. The accuracy of the numerical predictions which is very good up to incipient breaking, worse after wave splash-up events. This is mainly due to the fragmentation of the free surface that, in the realty, results in large inclusions of air beneath the maximum water level that persists possibly up the shoreline. Moreover, even if the width of the real tank is rather small, the experiments are not completely 2D as instead the SPH model. Since high turbulent breaking has intrinsic three-dimensional characteristics some small discrepancies between experimental and numerical results can be related to such stochastic phenomena. Future studies should consider different metrics for the identification of the free surface height at a longitudinal location in presence of a foamy air/water flow mixture. This may require the need of possibly solving the air flow too.

\section{Acknowledgments}

Authors wish to acknowledge Oliver Kimmoun and Hubert Branger for kindly share the set of high quality experimental measurements for the validation study and for the permission to reproduce their results. Authors also acknowledge the CINECA super-computing facility that, in the framework of the authors project ORPHEUS, made available the HPC Cluster Galileo where all the simulations have been run.

\section{References}

Ahrens, J.P., Titus, M.F., 1985. Wave runup formulas for smooth slopes. Journal of Waterway, Port, Coastal, and Ocean Engineering $111,128-133$.

Altomare, C., Crespo, A.J., Rogers, B., Dominguez, J., Gironella, X., Gomez-Gesteira, M., 2014. Numerical modelling of armour block sea breakwater with smoothed particle hydrodynamics. Computers and Structures 130, 34-45.

Altomare, C., Dominguez, J., Crespo, A., Gonzalez-Cao, J., Suzuki, T., Gomez-Gesteira, M., Troch, P., 2017. Long-crested wave generation and absorption for sph-based dualsphysics model. Coastal Engineering 127, 37-54.

Altomare, C., Dominguez, J., Crespo, A., Suzuki, T., Caceres, I., Gomez-Gesteira, M., 2015. Hybridization of the wave propagation model swash and the meshfree particle method sph for real coastal applications. Coastal Engineering Journal 57(4).

Antuono, M., Colagrossi, A., Marrone, S., Lugni, C., 2011. Propagation of gravity waves through an sph scheme with numerical diffusive terms. Computer Physics Communications 182, 866-877.

Batchelor, G.K., 2000. An introduction to fluid dynamics. Cambridge university press.

Battjes, J.A., 1975. Surf similarity, in: Coastal Engineering. American Society of Civil Engineers. volume 1, pp. 466-480.

Bayon, A., Valero, D., Garcia-Bartual, R., Lopez-Jimenez, P.A., et al., 2016. Performance assessment of openfoam and flow-3d in the numerical modeling of a low reynolds number hydraulic jump. Environmental Modelling and Software 80, 322-335. 
Bingham, H.B., Madsen, P.A., Fuhrman, D.R., 2009. Velocity potential formulations of highly accurate boussinesq-type models. Coastal Engineering 56, 467-478.

Chue, S., 1980. Wave run-up formula of universal applicability. Proceedings of the Institution of Civil Engineers 69, 1035-1041.

Crespo, A., Gomez-Gesteira, M., Dalrymple, R.A., 2007. Boundary conditions generated by dynamic particles in sph methods. CMCTECH SCIENCE PRESS- 5, 173.

Crespo, A.J., Dominguez, J.M., Rogers, B.D., Gomez-Gesteira, M., Longshaw, S., Canelas, R., Vacondio, R., Barreiro, A., GarciaFeal, O., 2015. Dualsphysics: Open-source parallel cfd solver based on smoothed particle hydrodynamics (sph). Computer Physics Communications 187, 204-216.

De Chowdhury, S., Anand, K., Sannasiraj, S., Sundar, V., 2017. Nonlinear wave interaction with curved front seawalls. Ocean Engineering 140, 84-96.

De Chowdhury, S., Sannasiraj, S., 2013. Sph simulation of shallow water wavepropagation. Ocean Engineering 60, 41-52.

De Leffe, M., Le Touze, D., Alessandrini, B., 2010. Sph modeling of shallow-water coastal flows. Journal of Hydraulic Research 48, $118-125$.

Dean, R., 1974. Evaluation and development of water wave theories for engineering application, vol 1 and 2. Special report nr 1 .

Didier, E., Neves, D., Martins, R., Neves, M., 2014. Wave interaction with a vertical wall: Sph numerical and experimental modeling. Ocean Engineering 88, 330-341.

FEMA, 1981. Manual for wave runup analaysis, coastal flood insurance studies. Stone and Webster Engrg. Corp., Boston , 96.

Galvin Jr, C.J., 1968. Breaker type classification on three laboratory beaches. Journal of geophysical research 73, 3651-3659.

Gomez-Gesteira, M., Cerqueiro, D., Crespo, C., Dalrymple, R., 2005. Green water overtopping analyzed with a sph model. Ocean Engineering 32, 223-238.

Gomez-Gesteira, M., Rogers, B.D., Crespo, A.J., Dalrymple, R.A Narayanaswamy, M., Dominguez, J.M., 2012. Sphysicsdevelopment of a free-surface fluid solver-part 1: Theory and formulations. Computers and Geosciences 48, 289-299.

Granthem, K.N., 1953. A model study of wave run-up on sloping structures - Series 3, Issue 348. Technical Report. CALIFORNIA UNIV. BERKELEY WAVE RESEARCH LAB.

Higuera, P., Lara, J.L., Losada, I.J., 2013a. Realistic wave generation and active wave absorption for navier-stokes models: Application to openfoam $\AA$. Coastal Engineering 71, 102-118.

Higuera, P., Lara, J.L., Losada, I.J., 2013b. Simulating coastal engineering processes with openfoam $\AA$. Coastal Engineering 71, 119-134.

Hsu, T.W., Liang, S.J., Young, B.D., Ou, S.H., 2012. Nonlinear runups of regular waves on sloping structures. Natural Hazards and Earth System Sciences 12, 3811-3820

Hughes, S.A., 2004. Estimation of wave run-up on smooth, impermeable slopes using the wave momentum flux parameter. Coastal Engineering 51, 1085-1104.

Hunt, I.A., 1959. Design of sea-walls and breakwaters. Transactions of the American Society of Civil Engineers 126, 542-570.

Iribarren, C., 1949. Protection des ports, in: XVIIth International Naval Congress (Lisbon, Portugal), 1949, pp. 31-80.

Khayyer, A., Gotoh, H., Shao, S., 2008. Corrected incompressible sph method for accurate water-surface tracking in breaking waves. Coastal Engineering 55, 236-250.

Kimmoun, O., Branger, H., 2007. A particle image velocimetry investigation on laboratory surf-zone breaking waves over a sloping beach. Journal of Fluid Mechanics 588, 353-397.

Kirby, J.T., 2016. Boussinesq models and their application to coastal processes across a wide range of scales. Ph.D. thesis. American Society of Civil Engineers.
Kobayashi, N., 1999. Wave runup and overtopping on beaches and coastal structures, in: Advances in coastal and ocean engineering. World Scientific, pp. 95-154.

Kobayashi, N., Otta, A.K., Roy, I., 1987. Wave reflection and run-up on rough slopes. Journal of Waterway, Port, Coastal, and Ocean Engineering 113, 282-298.

Landrini, M., Colagrossi, A., Greco, M., Tulin, M., 2007. Gridless simulations of splashing processes and near-shore bore propagation. Journal of Fluid Mechanics 591, 183-213.

Leimkuhler, B., Patrick, G.W., 1996. A symplectic integrator for riemannian manifolds. Journal of Nonlinear Science 6, 367-384.

Liu, X., Lin, P., Shao, S., 2015. Isph wave simulation by using an internal wave maker. Coastal Engineering 95, 160-170.

Losada, M.A., Gimenez-Curto, L.A., 1980. Flow characteristics on rough, permeable slopes under wave action. Coastal Engineering 4, 187-206.

Lubin, P., Glockner, S., 2015. Numerical simulations of threedimensional plunging breaking waves: generation and evolution of aerated vortex filaments. Journal of Fluid Mechanics 767, 364393.

Lubin, P., Glockner, S., Kimmoun, O., Branger, H., 2011. Numerical study of the hydrodynamics of regular waves breaking over a sloping beach. European Journal of Mechanics-B/Fluids 30, 552-564.

Madsen, P.A., Fuhrman, D.R., Wang, B., 2006. A boussinesq-type method for fully nonlinear waves interacting with a rapidly varying bathymetry. Coastal Engineering 53, 487-504.

Makris, C.V., Memos, C.D., Krestenitis, Y.N., 2016. Numerical modeling of surf zone dynamics under weakly plunging breakers with sph method. Ocean Modelling 98, 12-35.

Marrone, S., Colagrossi, A., Di Mascio, A., Le Touzé, D., 2016. Analysis of free-surface flows through energy considerations: Singlephase versus two-phase modeling. Physical Review E 93, 053113.

Martinez-Ferrer, P.J., Qian, L., Ma, Z., Causon, D.M., Mingham, C.G., 2018. Improved numerical wave generation for modelling ocean and coastal engineering problems. Ocean Engineering 152, 257-272.

Meringolo, D., Colagrossi, A., Marrone, S., Aristodemo, F., 2017. On the filtering of acoustic components in weakly-compressible sph simulations. Journal of Fluids and Structures 70, 1-23.

Monaghan, J., Kos, A., 1999. Solitary waves on a cretan beach. Journal of waterway, port, coastal, and ocean engineering 125, 145155.

Monaghan, J.J., 1992. Smoothed particle hydrodynamics. Annual review of astronomy and astrophysics 30, 543-574.

Monaghan, J.J., 2000. Sph without a tensile instability. Journal of Computational Physics 159, 290-311.

Monaghan, J.J., Lattanzio, J.C., 1985. A refined particle method for astrophysical problems. Astronomy and astrophysics 149, 135143.

Naeeja, M., Naeejb, M., Salehic, J., Rahimid, R., 2017. Modeling and evaluating of wave run-up and overtopping using smoothed particle hydrodynamics method. Computational Research Progress in Applied Science and Engineering 3, 25-34.

Nepf, H., Wu, C., Chan, E., 1998. A comparison of two-and threedimensional wave breaking. Journal of Physical Oceanography 28, 1496-1510.

Oh, S.H., Mizutani, N., Suh, K.D., Hashimoto, N., 2005. Experimental investigation of breaking criteria of deepwater wind waves under strong wind action. Applied Ocean Research 27, 235-250.

Rogers, B.D., Dalrymple, R.A., 2005. Sph modeling of breaking waves, in: Coastal Engineering 2004: (In 4 Volumes). World Scientific, pp. 415-427.

Rogers, B.D., Dalrymple, R.A., 2008. Sph modeling of tsunami waves, in: Advanced numerical models for simulating tsunami waves and runup. World Scientific, pp. 75-100. 
Roselli, R.A.R., Vernengo, G., Altomare, C., Brizzolara, S., Bonfiglio, L., Guercio, R., 2018. Ensuring numerical stability of wave propagation by tuning model parameters using genetic algorithms and response surface methods. Environmental Modelling and Software $103,62-73$.

Rota, A.R., Brizzolara, S., Chryssostomidis, C., Guercio, R., 2014 Numerical modeling of breaking periodical waves on a sloped beach profile by sph, in: The Twenty-fourth International Ocean and Polar Engineering Conference, International Society of Offshore and Polar Engineers.

Savage, R.P., 1959. Wave run-up on roughened and permeable slopes Transactions of the American Society of Civil Engineers 124, 852870 .

Saville, T., 1956. Wave run-up on shore structures. Journal of the Waterways and Harbors Division 82, 1-14.

Saville, T., 1958. Wave run-up on composited slopes, in: Proc. Conf. Coastal Eng., 6th, pp. 691-699.

Saville Jr, T., 1955. Laboratory Data on Wave Run-up and Overtopping on Shore Structures. Technical Report. COASTAL ENGINEERING RESEARCH CENTER VICKSBURG MS.

Schuttrumpf, H., 2001. Wellenuberlaufstromung an Seedeichen: Experimentelle und theoretische Untersuchungen. Ph.D. thesis.

Shao, S., Ji, C., 2006. Sph computation of plunging waves using a 2-d sub-particle scale (sps) turbulence model. International Journal for numerical methods in fluids 51, 913-936.

Shao, S., Ji, C., Graham, D.I., Reeve, D.E., James, P.W., Chadwick, A.J., 2006. Simulation of wave overtopping by an incompressible sph model. Coastal Engineering 53, 723-735.

Stoa, P., 1978. Reanalysis of wave runup on structures and beaches. technical paper no. 78-2. US Army, Corps of Engineers, Coastal Engineering Research Center. 131p .

Suzuki, T., Altomare, C., Veale, W., Verwaest, T., Trouw, K., Troch, P., Zijlema, M., 2017. Efficient and robust wave overtopping estimation for impermeable coastal structures in shallow foreshores using swash. Coastal Engineering 122, 108-123.

Thompson, A.C., 1989. Numerical model of breakwater wave flows, in: Coastal Engineering 1988, pp. 2013-2027.

Tian, Z., Perlin, M., Choi, W., 2008. Evaluation of a deep-water wave breaking criterion. Physics of Fluids 20, 066604.

Vacondio, R., Dal Palu, A., Mignosa, P., 2014. Gpu-enhanced finite volume shallow water solver for fast flood simulations. Environmental modelling and software 57, 60-75.

Valdez-Balderas, D., Dominguez, J.M., Rogers, B.D., Crespo, A.J., 2013. Towards accelerating smoothed particle hydrodynamics simulations for free-surface flows on multi-gpu clusters. Journal of Parallel and Distributed Computing 73, 1483-1493.

Violeau, D., 2012. Fluid mechanics and the SPH method: theory and applications. Oxford University Press.

Violeau, D., Leroy, A., 2015. Optimal time step for incompressible sph. Journal of Computational Physics 288, 119-130.

Weishar, L.L., Byrne, R.J., 1978. Field study of breaking wave characteristics, in: Coastal Engineering 1978, pp. 487-506.

Wu, C.H., Nepf, H., 2002. Breaking criteria and energy losses for three-dimensional wave breaking. Journal of Geophysical Research: Oceans 107.

Zijlema, M., Stelling, G., Smit, P., 2011. Swash: An operational public domain code for simulating wave fields and rapidly varied flows in coastal waters. Coastal Engineering 58, 992-1012. 